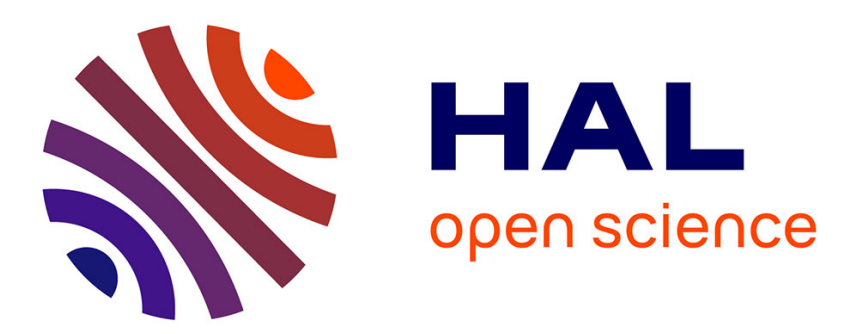

\title{
Electromagnetic Modeling of Complex Structures by TLM / Modal Hybrid Approach with Efficient Parallel Computing
}

Mihai Alexandru, Thierry Monteil, P Lorenz, Fabio Coccetti, Hervé Aubert

\section{- To cite this version:}

Mihai Alexandru, Thierry Monteil, P Lorenz, Fabio Coccetti, Hervé Aubert. Electromagnetic Modeling of Complex Structures by TLM / Modal Hybrid Approach with Efficient Parallel Computing. European Microwave Conference (EuMC), Oct 2012, Amsterdam, Netherlands. hal-01228328

\section{HAL Id: hal-01228328 \\ https://hal.science/hal-01228328}

Submitted on 16 Nov 2015

HAL is a multi-disciplinary open access archive for the deposit and dissemination of scientific research documents, whether they are published or not. The documents may come from teaching and research institutions in France or abroad, or from public or private research centers.
L'archive ouverte pluridisciplinaire HAL, est destinée au dépôt et à la diffusion de documents scientifiques de niveau recherche, publiés ou non, émanant des établissements d'enseignement et de recherche français ou étrangers, des laboratoires publics ou privés. 


\title{
Electromagnetic Modeling of Complex Structures by TLM / Modal Hybrid Approach with Efficient Parallel Computing
}

\author{
M. Alexandru* , T. Monteil", P. Lorenz ${ }^{+}$, F. Coccetti ${ }^{*}$ and H. Aubert* \\ *CNRS; LAAS; 7 avenue du Colonel Roche, F-31077 Toulouse, France \\ *Université de Toulouse; UPS, INSA, INP, ISAE; LAAS; F-31077 Toulouse, France \\ ${ }^{+}$Lorenz Solutions; 83661 Lenggries, Germany \\ mihai.alexandru@laas.fr
}

\begin{abstract}
This paper deals with the electromagnetic modeling of oversized and complex electrical structures by means of large scale parallel systems, such as the Grid Computing (GC). As numerical tools, a hybrid Transmission-Line Matrix (TLM) modeling method and modal approach is applied. The non-homogeneous volumes are discretized upon TLM, while the planar structures are modelled with the mode matching approach. The results prove the benefits of the combined GC and hybrid approach to solve electrically large structures.

Index Terms-Transmission line matrix methods, mode matching methods, grid computing, high performance computing, large-scale systems.
\end{abstract}

\section{INTRODUCTION}

One of the main challenge in nowadays Information Communications Technology (ICT) is to create smaller systems embedding more and more intelligence at hardware and software level with increasingly complex communicating architectures. These latter typically require robust design methodologies to reduce the development cycle and the prototyping phase. The complexity of these systems makes their optimization difficult mainly because of the explosion in the number of unknown parameters. In this context, the design and optimization of the communication physical layer is paramount.

The communicating objects are often integrated into cluttered environments with different metallic and dielectric structures, of larger or smaller sizes compared to the wavelength. The designer must anticipate the presence of such obstacles in the propagation channel to establish correct link budgets and a realistic design of the communicating object.

This paper presents a numerical tool for the rigorous calculation of the electromagnetic scattering phenomena inside supersized structures such as tunels, airplane cockpit and fuselage, containing large electrical size and complex patterns. A conventional 3D full-wave electromagnetic simulation would require enormous amount of computational resources.

So, in order to avoid that, the numerical method involved in this analysis is a time-domain hybrid based on computing domain decomposition as diakoptics approach. TLM models the electromagnetic field inside the non-homogeneous volumes, while the modal approach determines the electromagnetic field on the multiscale planar structures. Since this modeling approach involves the numerical solution of very large systems which is not easily accessible by traditional resources, the resolution is based on large scale parallel computing systems such as a computing grid. A general view over the presented approach is shown in Fig. 1.

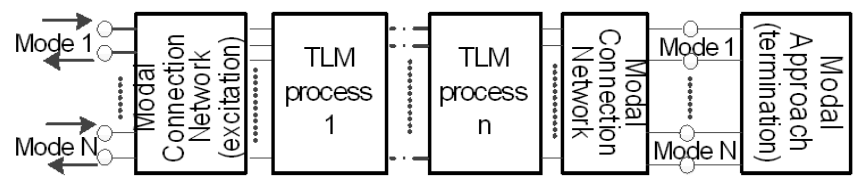

Fig. 1. Schematic view of the modeling approach.

A Grid-enabled time domain TLM system for full-wave analysis of complex electromagnetic structures is presented in [1]. A similar hybrid tool, modeling the scattering phenomena by diakoptics procedure, is in [2] and [3], where the discontinuity is discretized upon TLM and the homogeneous space by modal approach, given the low computing resources. In [4], the compromise done is reducing the TLM discretized space by setting the modal absorbing boundaries a few cells away from the discontinuities and considering only the first 4 or 5 modes. A numerical approach, based on an asymptotic code and a PWB method, to evaluate the high frequency coupling in a complex oversized structure is described in [5].

The example considered in the present work is a large waveguide discretized upon TLM - Symmetrical Condensed Nodes (SCNs), terminated in a homogeneous load (shortcircuit) or in a non-homogeneous load (inductive strip), modelled as a multi-port surface impedance by modal approach.

In Section II the hybrid numerical method approach between TLM and Mode Matching involved in our work is defined. Moreover the adaptation of the algorithm to be executed on Grid5000 platform [6] - the French national research infrastructure for large scale parallel and distributed computing, is introduced and described. The simulations done and the results derived are outlined in Section III.

\section{TLM / MODAL HYBRID APPROACH AND PARALLELIZATION}

The hybrid approach presented in this paper is validated by the electromagnetic simulation of the $T E_{10}$ mode propagation inside a lossless non-homogeneous rectangular waveguide. The volume structure is discretized upon TLM-SCN nodes with 18 ports for modeling the dielectric medium by stubs [7]. 
The boundaries of the discretized domain are two reference planes called Modal Connection Network, representing a matrix of a connection network based on ideal transformers, that permits us modeling the modal sources across the TLM cells on these planes [8]. As its ports have the same characteristic impedance - the free space impedance, as the TLM connecting-lines - the scattering matrix of the connection network is symmetric and unitary, which makes that the connection network and the TLM cell scattering matrix work well together. A Gaussian pulse excites the network of transmission lines on the excitation plan through wave digital filters.

A homogeneous load is modeled by the amplitude of the reflected mode obtained as a convolution product between the modal impulse response of the discontinuity and the amplitude of the incident mode.

A non-homogeneous load, as a symmetric narrow inductive strip, excites an infinite number of $T E_{2 n+10}$ modes $(\mathrm{n}=0,1,2, \ldots)$, propagating and evanescent, that couple each other. The multi-modal surface admittance matrix of the nonhomogeneous load is given by :

$$
\begin{gathered}
{[Y]=\frac{1}{\sum_{n=N+1}^{\infty} Z_{n}\left\langle\mathbf{g}_{e} \mid \mathbf{f}_{n}\right\rangle^{2}}[A][A]^{T}} \\
{[A]^{T}=\left[\left\langle\mathbf{g}_{e} \mid \mathbf{f}_{1}\right\rangle\left\langle\mathbf{g}_{e} \mid \mathbf{f}_{2}\right\rangle \cdots\left\langle\mathbf{g}_{e} \mid \mathbf{f}_{N}\right\rangle\right]}
\end{gathered}
$$

where, $N$ is the number of propagating modes, the sum refers to all evanescent modes, $Z_{n}$ is the impedance of the $n^{\text {th }}$ evanescent mode, $\boldsymbol{g}_{e}$ is an entire-domain trial function used in Galerkin's method for modeling the current density on the metallic strip, $\boldsymbol{f}_{n}$ is the $n^{\text {th }}$ basis function of the normal modal basis in the waveguide. The amplitude of the reflected modes is calculated similarly as in the case of homogeneous load.

In order to avoid a heavy TLM calculation, the discretized area is divided into subdomains that are calculated, in parallel, on different grid computing nodes that communicate between them to complete the job. The interaction between processes is achieved by an exchange of messages using Message-Passing Interface (MPI), a standard application programming interface for parallel implementations. So, all the tasks run the same program with different data.

A schematic view of the parallel hybrid approach implementation can be seen in Fig. 2. The user chooses the number of slices whose the structure to compute is decomposed. Each sub-domain is attributed to a process. On each process, the sub-structures are discretized and the boundary conditions are imposed. The excitation is performed only by the first task, while the termination is computed by the last process. At each time step, the TLM's core formed by the steps Scattering and Propagation is applied to the discretized cells. Before moving to the next time step, each process communicates to his neighboring processes the intermediate simulation data.

The speedup is a parameter used to evaluate the gain in terms of simulation time with $\mathrm{N}$ parallel computing processors compared to the same calculation performed on a single processor. The simulation time equals the sum between the

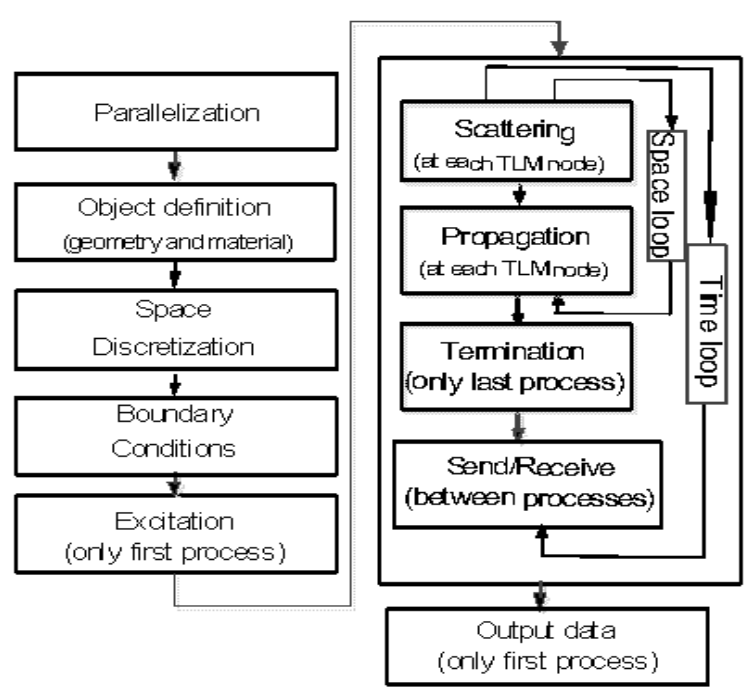

Fig. 2. Schematic view of the parallel hybrid approach implementation.

communication and computing time. The efficiency of the computation is the ratio between the speedup and the number of processes.

In order to estimate the computing time $T_{c a l}$, we define a prediction model according to the algorithm of the application:

$T_{c a l}=c_{1}+X Y c_{2}+t c_{3}+t X Y c_{4}+t X Z c_{5}+t Y Z c_{6}+t X Y Z c_{7}$,

where $X, Y, Z$ represent the number of TLM cells on the three carthesian directions, $t$ is the number of time steps and $c_{i}$, $i=1 . .7$ are the time coefficients corresponding to different blocks in the code of the application. The coefficients are determined using a linear programming formulation based on a history of experiments.

Considering a given structure $(\mathrm{X}, \mathrm{Y}, \mathrm{Z}, \mathrm{t})$, we can determine also the maximum number of processes $n$ required for computing the structure with the efficiency of at least $e$ :

$$
n \leq \frac{A+(1-e) B}{e\left(c_{1}+X Y c_{2}+t c_{3}+t X Y c_{4}+4 t T_{c o m}\right)},
$$

where:

$$
\begin{aligned}
& A=c_{1}+X Y c_{2}+t c_{3}+t X Y c_{4}, \\
& B=t X Z c_{5}+t Y Z c_{6}+t X Y Z c_{7}
\end{aligned}
$$

and $T_{\text {com }}$ is the communication time for one single sent message at a time step, the coefficient 4 represents the worst case, when a task sends and receives from the two neighboring processes. The communication time $T_{\text {com }}$ is defined by:

$$
T_{\text {com }}=l a t+m s g / d e b
$$

where lat is the network latency, msg represents the size of the sent message between two processes and $d e b$ is the network throughput. The size of the sent message depends on the number of TLM cells that are on the transversal surface.

\section{RESUlTS}

For a homogeneous load, the hybrid approach was validated by calculating the input Z-parameter - Fig. 3, of a lossless 
waveguide with the dimensions: $10 \mathrm{~mm}$ width, $5 \mathrm{~mm}$ height, $25 \mathrm{~mm}$ length and a mesh step of $1 \mathrm{~mm}$, filled with dielectric medium of $\epsilon_{r}=2.54$ and terminated on a short circuit. The input impedance computed with the hybrid approach follows the result obtained by High Frequency Structural Simulator (HFSS). While the simulation time with HFSS is about four minutes, the results of the TLM/modal hybrid approach are obtained in only 0.26 seconds with 2000 time steps, without graphical interface.

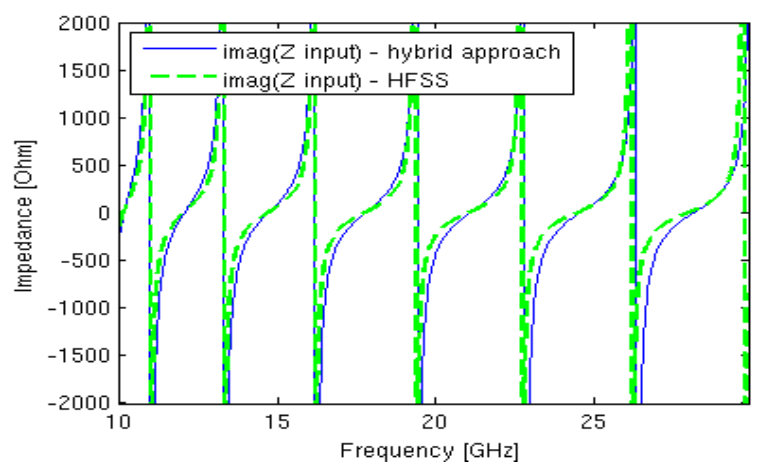

Fig. 3. Input impedance (imaginary part) of a lossless waveguide length filled with $\epsilon_{r}=2.54$ and terminated by a short-circuit.

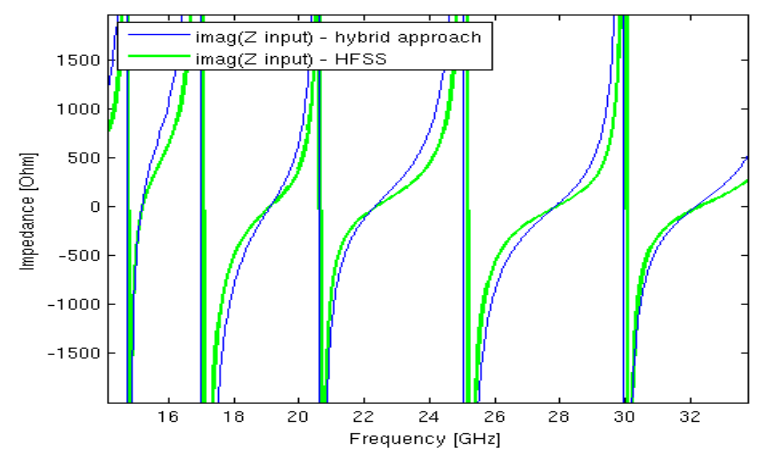

Fig. 4. Input impedance (imaginary part) of a lossless waveguide length filled with air and a block of dielectric $\epsilon_{r}=2.54$, and terminated by a short-circuit.

Also, the TLM/modal hybrid approach is validated for nonhomogeneous volumes by the simulation of a lossless short circuited waveguide, with the dimensions: $10 \mathrm{~mm}$ width, 5 $\mathrm{mm}$ height, $25 \mathrm{~mm}$ length and a mesh step of $1 \mathrm{~mm}$, filled with air and having a centered block of dielectric $\epsilon_{r}=2.54$, with the size of $4 \mathrm{~mm}$ width, $3 \mathrm{~mm}$ height, $3 \mathrm{~mm}$ length. In Fig. 4, the imaginary part of the input Z-parameter of this waveguide is in excellent agreement with the curve given by HFSS.

A length of lossless waveguide - Fig. 5 having the same dimensions as the waveguides mentioned above, with a centered block of dielectric $\epsilon_{r}=2.54$ and terminated by a metallic strip (1mm width) printed on a perfectly magnetic wall and modelled by (1), is computed by the hybrid approach. The TLM mesh step is of $0.2 \mathrm{~mm}$.

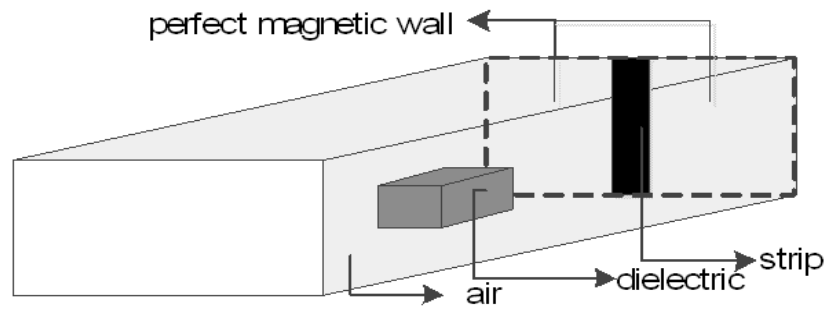

Fig. 5. Waveguide with dielectric bloc terminated on an inductive strip.
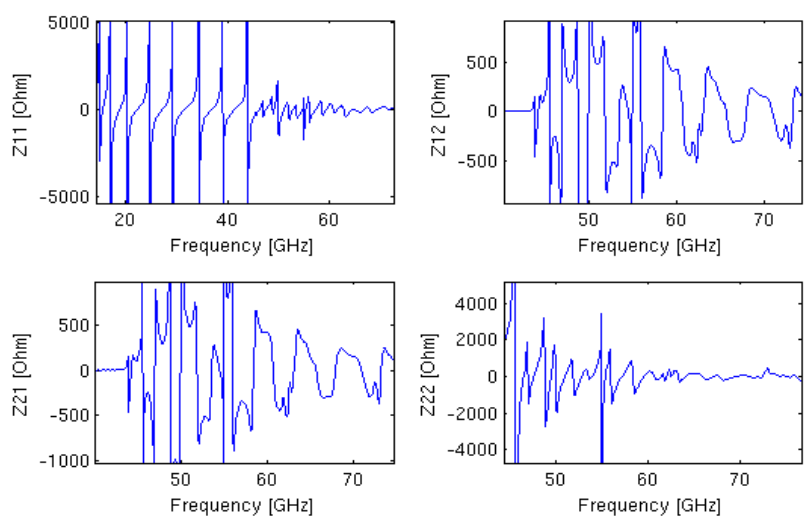

Fig. 6. Input impedance matrix elements (imaginary part) of a lossless waveguide length, filled with air and a dielectric block, terminated by a metallic strip printed on a perfectly magnetic wall (1 denotes the TE10 mode while 2 designates the TE30 mode).

We consider the excitation of the two first lower-order $T E$ propagating modes. The computed $2 \times 2$ input impedance matrix is presented in Fig. 6. The two modes excited by the inductive strip are coupling each other.

Simulations have been performed on grid environment to demonstrate the performance of the parallel hybrid approach presented in this paper. The computing nodes chosen are placed on four different clusters of Grid5000 (Griffon, Chinqchint, Paradent, Parapide)[9]. The clusters are linked by RENATER, the French network for research and teaching. Each computing node has two processors and each processor includes four cores. On a core runs only one process.

During several tests, we observed that the performance in terms of computing time drastically decreases when two or more processes run simultaneously on the same processor. In order to simulate as fast as possible, only two processes are executed on the computing node, each on a different processor.

Fig. 7 displays the speedup versus the number of processes, when a large waveguide $(345 \mathrm{~mm}$ width, $173 \mathrm{~mm}$ height, $2432 \mathrm{~mm}$ length and a mesh step of $1 \mathrm{~mm}$ ) is filled with vacuum and is terminated by a short circuit. As we increase the number of processes, the speedup values increase also, so the simulation time becomes smaller. The simulation time is about 46.13 hours using one process. With 256 processes, the structure is computed in only 0.74 hours, with an efficiency of $24 \%$. The predicted value given by (2), for the simulation 


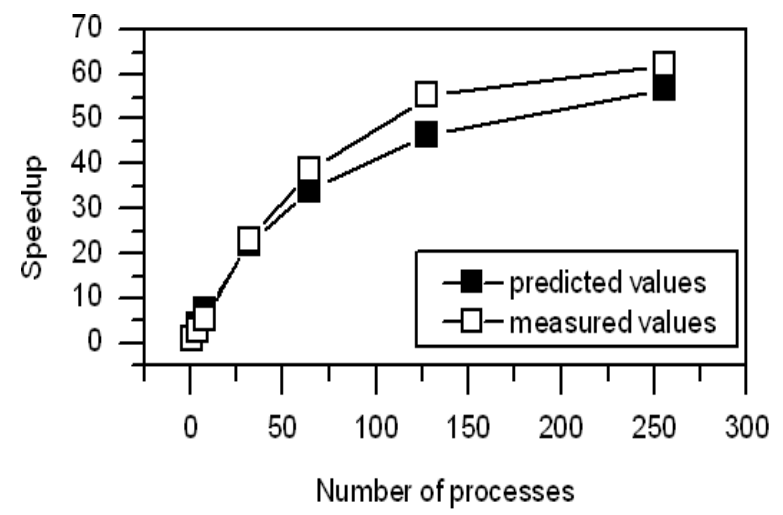

Fig. 7. Parallel computation Speedup for a waveguide modelled with 145 million of TLM cells.

time with one process, is 45.5 hours with an error of 1.41 . For the simulation with 256 processes, the time predicted value is 0.8 hours and the error, $7.4 \%$. In Fig. 7 , we can see also that the predicted speedup values for different number of processes follow the same evolution as the measured speedup values. The speedup curve shows the scalability of our hybrid approach as increasing the number of processes.

The prediction errors are caused by the cache misses that appear during the execution of the application, inside the TLM's core - Scattering and Propagation on Fig. 2 - at each time step.

However, as we increase the number of processes, the efficiency becomes smaller and the speedup tends to be invariant. Consequently, in order to launch efficient simulations we have to match the size of the structure with the number of resources used.

The number of processes that are necessary for calculation of our structure with the efficiency of $40 \%$, according to the prediction model given by (3) is 112 processes. The estimated value has an error of $14 \%$, compared to the measured value, 128 processes.

\section{CONCLUSION}

This paper presents an original approach which combines hybrid CEM technique with grid computing in order to speed up the modeling of large electromagnetic problems. The results displayed for the validation of the TLM/modal hybrid approach presented in this communication match very well the results given by HFSS. The study has highlighted the role of parallelization scheme on grid, with respect to the size of the problem and its repartition. The parallel hybrid approach permits us to model complex structures containing large electrical size and complex multiscale patterns. The computing time prediction model outlined in this work, (2), let us estimate the ressource reservation time to simulate a given structure on grid. Also, characterizing the performance of the simulations on the computing grid, rules for the estimation of the required resources have been given in (3).

In perspective, running simulations over several billion of TLM cells and developping a parallel hybrid numerical method coupling TLM to a frequency-domain method, as Scale Changing Technique (SCT) [10], represents the final goal toward fast and full-wave electromagnetic simulation of complex and electrically large structures.

\section{REFERENCES}

[1] P. Lorenz, J. Vital, B. Biscontini, and P. Russer, "A grid-enabled time domain transmission line matrix (tlm-g) system for the analysis of complex electromagnetic structures," in Workshop on Computational Electromagnetics in Time-Domain(CEM-TD), nov. 2005, pp. 48-51.

[2] M. Righi and W. Hoefer, "Efficient 3d-scn-tlm diakoptics for waveguide components," in Microwave Symposium Digest, 1994., IEEE MTT-S International, may 1994, pp. 27-30 vol.1.

[3] M. Righi, J. Herring, and W. Hoefer, "Efficient hybrid tlm/modematching analysis of packaged components," IEEE Trans. on Microwave Theory and Tech., vol. 45, no. 10, pp. 1715-1724, oct 1997.

[4] L. Pierantoni, C. Tomassoni, and T. Rozzi, "A new termination condition for the application of the tlm method to discontinuity problems in closed homogeneous waveguide," IEEE Trans. on Microwave Theory and Tech., vol. 50, no. 11, pp. 2513-2518, nov 2002.

[5] I. Junqua, J. Parmantier, and M. Ridel, "Modeling of high frequency coupling inside oversized structures by asymptotic and pwb methods," in International Conference on Electromagnetics in Advanced Applications (ICEAA), sept. 2011, pp. 68-71.

[6] F. Cappello, E. Caron, M. Dayde, F. Desprez, Y. Jegou, P. Primet, E. Jeannot, S. Lanteri, J. Leduc, N. Melab, G. Mornet, R. Namyst, B. Quetier, and O. Richard, "Grid'5000: a large scale and highly reconfigurable grid experimental testbed," in Grid Computing, 2005. The 6th IEEE/ACM International Workshop on, nov. 2005, p. 8 pp.

[7] P. Johns, "A symmetrical condensed node for the tlm method," IEEE Trans. on Microwave Theory and Tech., vol. 35, no. 4, pp. 370-377, apr 1987.

[8] P. Lorenz and P. Russer, "Discrete and modal source modeling with connection networks for the transmission line matrix (tlm) method," in Microwave Symposium, 2007. IEEE/MTT-S International, june 2007, pp. $1975-1978$

[9] Grid5000. (2012) Grid5000:hardware. [Online]. Available: https://www.grid5000.fr/mediawiki/index.php/Special:G5KHardware

[10] H. Aubert, "The concept of scale-changing network in global electromagnetic simulation of complex structures," Progress in Electromagnetics Research B, vol. 16, pp. 127-154, 2009. 